\title{
Neutrino Propagation and Oscillations in a Strong Magnetic Field*
}

\author{
Efrain J. Ferrer and Vivian de la Incera \\ Physics Department, State University of New York at Fredonia, \\ Fredonia, NY 14063, USA
}

\begin{abstract}
We review the results on neutrino propagation in neutral and charged media under strong magnetic fields $M_{W}^{2} \gg B \gg m_{e}^{2}$. It is shown that the neutrino energy density gets a magnetic contribution in both charged and neutral media, which is linear in the magnetic field, of first order in $G_{F}$, and independent of the charge density. This new term enters as a correction to the neutrino kinetic energy and produces an anisotropic contribution to the neutrino index of refraction. As a consequence, in a neutral medium a highly anisotropic resonant level-crossing condition takes place for the oscillation between electron-neutrinos and the other neutrino species. Possible cosmological applications are presented.
\end{abstract}

\section{Introduction}

In our universe the magnetic field is omnipresent. Large magnetic fields $B=$ $10^{12}-10^{14} G$ have been associated with the surface of supernovas [1] and neutron stars [2], and fields perhaps as large as $10^{16} G$ with magnetars [3]. Even larger fields could exist in the star's interior. Based on the scalar virial theorem [4], it has been suggested that the interior field in neutron stars could be as high as $10^{18} \mathrm{G}$. On the other hand, the early universe was presumably permeated by large primordial magnetic fields that eventually became the seeds [5] 6] of the galactic fields that are observed today at scales of $100 \mathrm{Kpc}$ and larger [7.

In cosmology, the effects of primordial magnetic fields on neutrino propagation are of special interest during the neutrino decoupling era, since in that epoch a large number of these particles escaped the electroweak plasma with the possibility to develop significant oscillations. As for astrophysics, the interconnection between a star's magnetic field and its particle current flow, is of great relevance to understand the star's evolution.

\footnotetext{
*Talk given at the Conference: MRST-2004: "From Quaks to Cosmology," Montreal, Canada, May 12-14, 2004
} 
Thus, a thorough study of the effects of strong magnetic fields on neutrino propagation is crucial for both astrophysics and cosmology. In this sense it should be noticed that unlike the stellar material, whose density can be of order one, the early universe was almost charge symmetric, with a particle-antiparticle asymmetry of only $10^{-9}-10^{-10}$. Therefore, when considering possible applications to the early universe, we should restrict the discussion to the effects on a magnetized neutral medium ( $\mu=0$, where $\mu$ is the chemical potential characterizing the system's unbalance between particles and antiparticles); while in the interior of neutron stars it is natural to consider a magnetized charged medium $(\mu \neq 0)$.

The propagation of neutrinos in magnetized media has been previously investigated by several authors [8-11]. The weak-field results of papers [8-11] led to think that the magnetic-field effects could be significant in astrophysics, because of the field- and $\mu$-dependent terms of order $1 / M_{W}^{2}$, but were irrelevant $\left(\sim 1 / M_{W}^{4}\right.$-order $)$ in the early universe due to its charge-symmetric character $(\mu=0)$. However, as we have recently found [12-15], and will discuss below, a strong magnetic field $\left(M_{W} \gg \sqrt{B} \gg m_{e}, T, \mu,|\mathbf{p}|\right)$ gives rise to a new contribution to the neutrino energy that is linear in the field, independent of the chemical potential, and that is of the same order $\left(1 / M_{W}^{2}\right)$ as the largest terms found in the weak-field charged-medium case. This new result turns magneticfield effects relevant for cosmology.

\section{Neutrino Self-Energy in Magnetized Media. Covariant Structure}

The neutrino self-energy operator $\sum(p)$ is a Lorentz scalar that can be formed in the spinorial space taking the contractions between the characteristic vectors and tensors of the system with all the independent elements of the Dirac ring. Explicit chirality reduces it to

$$
\sum=R \bar{\sum} L, \quad \bar{\sum}=V_{\mu} \gamma^{\mu}
$$

where $L, R=\frac{1}{2}\left(1 \pm \gamma_{5}\right)$ are the chiral projectors, and $V_{\mu}$ is a Lorentz vector that can be spanned as a superposition of four basic vectors formed with the characteristic tensors of the problem. In a magnetized medium, besides the neutrino four-momentum $p_{\mu}$, and the four-velocity of the center of mass of the magnetized medium $u_{\mu}$, we have to consider the magnetic-field strength tensor $F_{\mu \nu}$. In a neutral medium it is convenient to use the expansion

$$
\bar{\sum}(p, B)=a p_{\|}+b p_{\perp}+c p^{\mu} \widehat{\widetilde{F}}_{\mu \nu} \gamma^{\nu}+i d p^{\mu} \widehat{F}_{\mu \nu} \gamma^{\nu}
$$

where we introduced the notations $\widehat{F}_{\mu \nu}=\frac{1}{|B|} F_{\mu \nu}, \widehat{\widetilde{F}}_{\mu \nu}=\frac{1}{2|B|} \varepsilon_{\mu \nu \rho \lambda} F^{\rho \lambda}, \not p_{\|}=$ $p^{\mu} \widehat{\widetilde{F}}_{\mu}^{\rho} \widehat{\widetilde{F}}_{\rho \nu} \gamma^{\nu}$, and $p_{\perp}=p^{\mu} \widehat{F}_{\mu} \rho \widehat{F}_{\rho \nu} \gamma^{\nu}$. 
In a charged medium, it will be more convenient (the coefficients in this basis will be non-singular in the $p \rightarrow 0$ limit) to expand $\bar{\sum}$ in a different basis

$$
\bar{\sum}(p, B)=a^{\prime} p p+b^{\prime} \psi+c^{\prime} \widehat{B}+i d^{\prime} p^{\mu} \widehat{F}_{\mu \nu} \gamma^{\nu} .
$$

In the covariant representation the magnetic field is expressed as $B_{\mu}=$ $\frac{1}{2} \varepsilon_{\mu \nu \rho \lambda} u^{\nu} F^{\rho \lambda}$. The coefficients $a\left(a^{\prime}\right), b\left(b^{\prime}\right), c\left(c^{\prime}\right)$, and $d\left(d^{\prime}\right)$ are Lorentz scalars that depend on the parameters of the theory and the approximation used. Our goal is to find those coefficients in the one-loop approximation, and hence, the dispersion relations for different systems in the presence of strong magnetic-field backgrounds.

\section{$3 \quad$ Neutrino Dispersion Relation in Strongly Mag- netized Media}

The neutrino dispersion relation in a magnetic background can be found as the nontrivial solution of

$$
\operatorname{det}\left[p+\sum(p, B)\right]=0 .
$$

In the one-loop approximation, the neutrino self-energy is given by

$$
\Sigma(x, y)=\frac{i g^{2}}{2} R \gamma_{\nu} S(x, y) \gamma^{\mu} G_{F}(x, y)_{\mu}{ }^{\nu} L
$$

where $S(x, y)$ and $G_{F}(x, y)_{\nu}{ }^{\mu}$ are the Green's functions of the electron and $\mathrm{W}$-boson, in the presence of the magnetic field, respectively. Since the two virtual particles are electrically charged, the magnetic field interacts with both producing a Landau quantization of the corresponding transverse momenta [12$15]$.

In a medium the neutrino self-energy operator can be unfolded as

$$
\bar{\Sigma}(p, B)=\bar{\Sigma}_{0}(p, B)+\bar{\Sigma}_{T}(p, B),
$$

where $\bar{\Sigma}_{0}$ is the field-dependent vacuum contribution and $\bar{\Sigma}_{T}$ is the statistical part which is a function of the temperature and the magnetic field (in a charged medium the statistical part will also depend on the chemical potential and will be represented by $\left.\bar{\Sigma}_{T, \mu}(p, B)\right)$. Our interest is to find $\bar{\Sigma}(p, B)$ in the strong-field approximation defined by the condition $M_{W} \gg \sqrt{B} \gg m_{e}, T,|\mathbf{p}|$. Since in this approximation the gap between the electron Landau levels is larger than the rest of the parameters entering in the electron energy, it is consistent to use the lowest Landau level (LLL) approximation in the electron Green's function, while in the W-boson Green's function, because $M_{W} \gg \sqrt{B}$, we must sum in all W-boson Landau levels. 


\subsection{Neutral Case}

Within the strong field approximation, the vacuum and statistical parts of the neutrino self-energy in a magnetized neutral medium were found in Refs. [12], and [15] respectively. Up to leading order in powers of $1 / M_{W}^{2}$ it is given by

$$
\begin{gathered}
\bar{\Sigma}_{T}(p, B)=a p_{\|}+c p^{\mu} \widehat{\widetilde{F}}_{\mu \nu} \gamma^{\nu}, \\
a=-c=\frac{-g^{2} e B}{8 M_{W}^{2}}\left[\frac{1}{(2 \pi)^{2}}+\frac{T^{2}}{3 M_{W}^{2}}\right] \exp \left(-p_{\perp}^{2} / e B\right) .
\end{gathered}
$$

Solving the dispersion equation (44) for the self-energy (7) we obtain the neutrino energy spectrum in the strongly magnetized neutral medium as

$$
E_{ \pm}=|\mathbf{p}|\left[1-a \sin ^{2} \alpha\right]
$$

In (9), $\alpha$ is the angle between the direction of the neutrino momentum and that of the applied magnetic field, and $E_{ \pm} \equiv \pm p_{0}$ are the neutrino $(+) /$ anti-neutrino (-) energies, respectively.

From (8) it is clear that in the strong-field limit the thermal correction to the neutrino energy is much smaller $\left(\sim 1 / M_{W}^{2}\right.$ smaller $)$ than the vacuum correction. This result can be significant for cosmological applications in case a strong primordial magnetic field were present in the primeval plasma. The neutrino energy spectrum (9) depends on the neutrino direction of motion, with a maximum field effect for neutrinos propagating perpendicularly to the magnetic field.

\subsection{Charged Case}

We consider now a medium with a finite density of electric charge. As usual, a finite density is reflected through the introduction of a chemical potential $\mu$, which plays the role of an external parameter. Here the following comment is in order, in electroweak matter at finite density there exists the possibility to induce additional "chemical potentials" 16. These chemical potentials are nothing but dynamically generated background fields given by the average $\left\langle W_{0}^{3}\right\rangle$ and $\left\langle B_{0}\right\rangle$ of the zero components of the gauge fields. They are known to appear, for instance, in the presence of finite lepton/baryon density [16] (for recent applications related to this effect see Refs. [17]). In our case, a possible consequence of the condensation of such average fields could be the modification of the effective chemical potential of the W-boson, which then might be different from the electron chemical potential. Nevertheless, that modification, even if present in the case under study, will have no relevant consequences, as the W-boson contribution will always be suppressed by the factor $e^{-M_{W} / T}$.

We find [15] that the leading contribution to the neutrino self-energy in a strongly magnetized charged medium is

$$
\bar{\Sigma}_{T, \mu}(p, B)=b^{\prime} \not+c^{\prime} \widehat{B}
$$




$$
b^{\prime}=-c^{\prime}=\frac{g^{2} e B \mu}{2(4 \pi)^{2} M_{W}^{2}} e^{-p_{\perp}^{2} / 2 e B}\left[p_{0}-p_{3}-4 \mu\right]
$$

Using the result (10) to solve the dispersion relation (4), we find that the neutrino energy spectrum in the strongly magnetized charged medium is given in leading order in the Fermi coupling constant by

$$
E_{ \pm}=|\mathbf{p}|\left[1-a(T=0) \sin ^{2} \alpha\right]-\mathcal{M} \cdot \mathbf{B} \pm E_{\mathrm{o}}
$$

where

$$
\mathcal{M} \equiv \frac{-E_{\mathrm{o}}}{|\mathbf{B}|} \frac{\mathbf{p}}{|\mathbf{p}|}, \quad E_{0}=\sqrt{2} G_{F} e^{-p_{\perp}^{2} / 2 e B}\left[N_{0}^{-}-N_{0}^{+}\right] .
$$

and we introduced the notation $N_{0}^{\mp}$ for the electron/positron number densities in the LLL.

In the RHS of Eq. (12), the first term is the modified neutrino kinetic energy, with the field-dependent radiative correction, $a(T=0)$, obtained after taking $T=0$ in Eq. (8); the second term can be interpreted as a magneticmoment/magnetic-field interaction energy, with $\mathcal{M}$ playing the role of a neutrino effective magnetic moment; and the third term is a rest energy induced by the magnetized charged medium. In the case under study we have that although the neutrino is a neutral massless particle, the charged medium can endow it, through quantum corrections, with a magnetic moment proportional to the induced neutrino rest energy.

In a charged medium CP-symmetry is violated [18. A common property of electroweak media with CP violation is the separation between neutrino and anti-neutrino energies. In the present case this property is manifested in the double sign of $E_{0}$ in (12).

\section{Neutrino Oscillations in a Strongly Magne- tized Primeval Plasma}

The strength of the primordial magnetic field in the neutrino decoupling era can be estimated from the constraints derived from the successful nucleosynthesis prediction of primordial ${ }^{4} \mathrm{He}$ abundance [19], as well as from neutrino mass and oscillation limits 20]. These constraints, together with the energy equipartition principle, lead to the relations

$$
m_{e}^{2} \leq e B \leq m_{\mu}^{2}, \quad B / T^{2} \sim 2,
$$

with $m_{\mu}$ being the muon mass. It is reasonable to assume the existence of a primordial magnetic field in the above range in the epoch between the QCD phase transition and the end of nucleosynthesis [12.

Notice that a field satisfying (14) would be effectively strong with respect to the electron-neutrino, but weak for the remaining neutrino flavors. If such a field existed during the decoupling era, it could significantly affect the $\nu_{e} \leftrightarrow \nu_{\mu}, \nu_{\tau}$ 
and $\nu_{e} \leftrightarrow \nu_{s}$ resonant oscillations [14, as the field would differently modify the energy of $\nu_{e}$ compared to those of $\nu_{\mu}, \nu_{\tau}$ and $\nu_{s}$.

Let us consider the evolution equation in the presence of a strong magnetic field for a two-level system

$$
\frac{d}{d t}\left(\begin{array}{c}
\nu_{e} \\
\nu_{\mu}
\end{array}\right)=H_{B}\left(\begin{array}{c}
\nu_{e} \\
\nu_{\mu}
\end{array}\right)
$$

where the Hamiltonian $H_{B}$ is given by

$$
H_{B}=p+\frac{m_{1}^{2}+m_{2}^{2}}{4 p}+\left(\begin{array}{ll}
-\frac{\Delta m^{2}}{4 p} \cos 2 \theta+E_{ \pm} & \frac{\Delta m^{2}}{4 p} \sin 2 \theta \\
\frac{\Delta m^{2}}{4 p} \sin 2 \theta & \frac{\Delta m^{2}}{4 p} \cos 2 \theta
\end{array}\right)
$$

Here, $\theta$ is the vacuum mixing angle, $\Delta m^{2}=m_{2}^{2}-m_{1}^{2}$ is the mass square difference of the two mass eigenstates, and the magnetic energy density contribution to the electron-neutrino $E_{ \pm}$is given in Eq. (9).

In Eq. (16) the magnetic field contribution to the muon-neutrino in the second diagonal term has been neglected, taking into account that it will be of second order in the Fermi coupling constant as corresponds to the weak-field approximation in the neutral medium.

If we consider that the only flavor present at the initial time was the electronneutrino, using the evolution equation (15)-(16) we find that the appearance probability for the muon neutrino is given by

$$
P_{B}\left(\nu_{e} \rightarrow \nu_{\mu}\right)=\sin ^{2} \theta_{B} \sin ^{2} \frac{\pi x}{\lambda}
$$

where $\lambda$ is the oscillation length in the magnetized space

$$
\lambda=\frac{\lambda_{0}}{\left[\sin ^{2} 2 \theta+\left(\cos 2 \theta-\frac{\lambda_{0}}{\lambda_{e}}\right)\right]^{1 / 2}}
$$

and

$$
\sin ^{2} 2 \theta_{B}=\frac{\sin ^{2} 2 \theta}{\left(\cos 2 \theta-\frac{\lambda_{0}}{\lambda_{e}}\right)^{2}+\sin ^{2} 2 \theta}
$$

is the probability amplitude written in terms of the vacuum $\left(\lambda_{0}=\frac{4 \pi p}{\Delta m^{2}}\right)$ and magnetic $\left(\lambda_{e}=\frac{2 \pi}{E_{ \pm}}\right)$oscillation lengths.

If the resonant condition

$$
\frac{\lambda_{0}}{\lambda_{e}}=\cos 2 \theta
$$

is satisfied, then the probability amplitude (19) will get is maximum value independently of the value of the mixing angle in vacuum $\theta$ (the same effect will be obtained between electron-neutrinos and tau or sterile neutrinos). The condition (20) is a resonant level-crossing condition. We underline that the resonant effect in the magnetized neutral medium is anisotropic, as it depends on the 
direction of propagation of the electron-neutrino with respect to the magnetic field (see that $\lambda_{e}$ depends on $\alpha$ ).

The resonant phenomenon here is similar to that in the well known MSW effect [21] in a charged medium. Nevertheless, we stress that in the magnetized neutral medium the oscillation process does not differentiate between neutrinos and antineutrinos.

\section{Concluding Remarks}

A main outcome of our investigation was to show that in strongly magnetized systems a term of different nature emerges in both charged and neutral media. The new term, which is linear in the magnetic field and of first order in $G_{F}$, enters as a correction to the neutrino kinetic energy. This correction is present even in a strongly magnetized vacuum [12, since it is related to the vacuum part $(T=0, \mu=0)$ of the neutrino self-energy at $B \neq 0$. A characteristic of the field-dependent corrections to the neutrino energy is that they produce an anisotropic index of refraction. We call attention that the anisotropy does not differentiate between neutrinos and antineutrinos.

The charged-medium results reported in the current work can be of interest for the astrophysics of neutrinos in stars with large magnetic fields. On the other hand, our finding for the neutral medium can have applications in cosmology, if the existence of high primordial magnetic fields is finally confirmed. Contrary to some authors' belief [10, that regardless of the field intensity, the neutrino dispersion relation in the early universe is well approximated by the dispersion relation in the zero field medium, our results indicate that if strong, and even weakly-strong [15] magnetic fields existed in the neutrino decoupling era, they could have an impact in neutrino flavor oscillations in the primeval plasma.

\section{Acknowledgment}

This work was supported by NSF-grant PHY-0070986

\section{References}

[1] V. Ginzburg, High Energy Gamma Ray Astrophysics (North-Holland, Amsterdam, 1991); D. Bhattacharya and G. Srinivasan, in X-ray Binaries, ed. W.H.G. Lewin, J. van Paradijs, and E.P.J. van den Heuvel, Cambridge Univ. Press, Cambridge, 1995, pag. 495.

[2] I. Fushiki, E. H. Gudmundsson, and C.J. Pethick, Astrophys. J.342, 958 (1989); T.A. Mihara, et. al., Nature (London) 346, 250 (1990); G. Chanmugam, Annu. Rev. Astron. Astrophys. 30, 143 (1992). 
[3] R. C. Duncan and C. Thompson, Astrophys. J. 392 (1992) L9; B. Paczynski, Acta Astron. 42, 145 (1992); V. V. Usov, Nature 357, 472 (1992); C. Thompson and R. C. Duncan Astrophys. J. 473, 322 (1996); C. Kouveliotou et al., Nature 393, 235 (1998); Astrophys. J. 510, L115 (1999).

[4] D. Lai and S.L. Shapiro, Astrophys. J. 383, 745 (1991) and references therein.

[5] D. Grasso and H.R. Rubinstein, Phys. Rep. 348, 163 (2001).

[6] K. Enqvist, Int. J. Mod. Phys. D7, 331 (1998).

[7] Y. Sofue, M. Fujimoto, and R. Wielebinski, Ann. Rev. Astron. Astrophys. 24, 459 (1986); P. P. Kronberg, Rep. Prog. Phys. 57, 325 (1994); R. Beck et. al., Ann. Rev. Astron. Astrophys. 34, 153 (1996); R. Beck et. al., Ann. Rev. Astron. Astrophys. 34, 153 (1996).

[8] G. McKeon, Phys. Rev. D24, 2744 (1981).

[9] A. Erdas, and G. Feldman, Nucl. Phys. B343, 597 (1990).

[10] V. B. Semikoz and J. W. F. Valle, Nucl. Phys. B425, 651 (1994); P. Elmfors, D. Grasso and G. Raffelt, Nucl. Phys. B479, 3 (1996); A. Erdas, C. W. Kim and T. H. Lee, Phys. Rev. D58, 085016 (1998); D. Grasso, Nucl. Phys. B (Proc. Suppl.) 70, 267 (1999); A. Erdas, and C. Isola, Phys. Lett. B494, 262 (2000).

[11] J. C. D'Olivo, J. F. Nieves and P. B. Pal, Phys. Rev. D40, 3679 (1989).

[12] E. Elizalde, E. J. Ferrer and V. de la Incera, Ann. of Phys. 295, 33 (2002).

[13] E. J. Ferrer, in Proceedings of the International Conference "Quantization, Gauge Theory, And Strings," 5-10 Jun 2000, Moscow, Russia (Scientif World Publi., Singapore 2001, V. II, pag.301, editors A. Semikhatov, M. Vasiliev and V. Zaikin); E. Elizalde, E. J. Ferrer and V. de la Incera, Neutrino Dispersion in Intense Magnetic Field. Proceedings of Particle Physics and Cosmology Second Tropical Workshop (AIP Conference Proceedings V540, pag. 372, editor J. F. Nieves), San Juan, Puerto Rico, Apr. 2000.

[14] E. J. Ferrer and V. de la Incera, Neutrinos under Strong Magnetic Fields. Proceedings of Neutrinos, Flavor Physics and Precision Cosmology, Fourth Tropical Workshop (AIP Conference Proceedings V689, pag. 372, editor J. F. Nieves and R. R. Volkas), Cairns, Queensland, Australia, June 2003.

[15] E. Elizalde, E. J. Ferrer and V. de la Incera, hep-ph/0404234 (To appear in Phys. Rev. D70 ).

[16] A. D. Linde, Phys. Lett. B86, 39 (1979); E. J. Ferrer, V. Incera and A. E. Shabad, Phys. Lett. B185, 407 (1987); Nucl. Phys. B309, 120 (1988). 
[17] A. Gynther, Phys. Rev. D68, 016001 (2003); F. Sannino and K. Tuominen, Phys. Rev. D68, 016007 (2003); V. P. Gusynin, V. A. Miransky and I. Shovkovy, Phys. Lett. B58, 82 (2004); Mod. Phys. Lett. A19, 1341 (2004).

[18] P. B. Pal and T. N. Pham, Phys. Rev. D40, 259 (1989); J. F. Nieves, Phys. Rev. D40, 866 (1989).

[19] K. A. Olive, D. N. Schramm, G. Steigman, and T. Walker, Phys. Lett. B236, 454 (1990); L. M. Krauss and P. Romanelli, Astrophys. J. 358, 47 (1990).

[20] K. Enqvist, V. Semikoz, A. Shukurov, and D. Sokoloff, Phys. Rev. D48, 4557 (1993).

[21] L. Wolfenstein, Phys. Rev. D17, 2369 (1978); S. P. Mkheyev, A. Yu. Smirnov, Sov. J. Nucl. Phys. 42, 913 (1985). 